\title{
A supergeneralização da memória autobiográfica nos transtornos depressivos
}

\author{
Overgeneral autobiographical memory in depressive disorders
}

Tarcísio Gomes Dutra ${ }^{1}$, Laila da Camara Lima Kurtinaitis ${ }^{1}$, Amaury Cantilino ${ }^{2}$, Maria Carolina Souto de Vasconcelos ${ }^{3}$, Izabel Hazin ${ }^{4}$, Everton Botelho Sougey ${ }^{5}$

\section{Resumo}

Este artigo tem como objetivo revisar estudos que possuíram como temática a relação entre o fenômeno da supergeneralização da memória autobiográfica e os transtornos depressivos. Tal característica tem chamado a atenção por sua relação com uma pobre habilidade de resolução de problemas e imaginação do futuro, bem como pela manutenção e pior prognóstico da depressão. Para o acesso das informações, foi realizada uma busca sistematizada nos bancos de dados LILACS, SciELO, MEDLINE, IBECS e nas bases de ciências da saúde do Portal de Periódicos da Coordenação de Aperfeiçoamento de Pessoal de Nível Superior (CAPES), com foco em artigos publicados entre 2000 e 2010, usando os seguintes descritores: memória autobiográfica, supergeneralização da memória autobiográfica e memória autobiográfica e depressão, em português; e autobiographical memory, overgeneral autobiographical memory e autobiographical memory and depression em inglês. Após a aplicação dos critérios de exclusão, 27 estudos foram revisados. A supergeneralização tem sido investigada em diversos transtornos depressivos. Entretanto, mais estudos longitudinais são necessários para fundamentar tal característica cognitiva como um aspecto relevante na anamnese e no tratamento de distúrbios do humor.

Descritores: Memória autobiográfica, depressão, cognição.

\begin{abstract}
This article aims to review studies focusing on the relationship between overgeneral autobiographical memory and depressive disorders. Such characteristic has attracted attention because of its relationship with a poor ability to solve problems and to imagine the future, as well as with the maintenance and a poor prognosis of depression. Data were collected through a systematic search on LILACS, SciELO, MEDLINE, and IBECS databases, and also on the health sciences records of Portal de Periódicos da Coordenação de Aperfeiçoamento de Pessoal de Nível Superior (CAPES), a Brazilian journal database, focusing on articles published between 2000 and 2010. The following keywords were used: memória autobiográfica, supergeneralização da memória autobiográfica, and memória autobiográfica e depressão in Portuguese; and autobiographical memory, overgeneral autobiographical memory, and autobiographical memory and depression in English. Following application of exclusion criteria, a total of 27 studies were reviewed. Overgeneral autobiographical memory has been investigated in several depressive disorders. However, further longitudinal studies are required to confirm the relevant role of this cognitive characteristic in anamnesis and in the treatment of mood disorders.
\end{abstract}

Keywords: Autobiographical memory, depression, cognition.

\footnotetext{
${ }^{1}$ Psicólogo(a). Mestre, Neuropsiquiatria e Ciências do Comportamento, Universidade Federal de Pernambuco (UFPE), Recife, PE. ${ }^{2}$ Psiquiatra. Doutor. Coordenador, Programa de Saúde Mental da Mulher, UFPE. ${ }^{3}$ Acadêmica de Psicologia, UFPE. ${ }^{4}$ Psicóloga. Pós-doutora. Professora adjunta, Departamento de Psicologia, Universidade Federal do Rio Grande do Norte (UFRN), Natal, RN. ${ }^{5}$ Psiquiatra. Pós-doutor. Professor associado, Departamento de Neuropsiquiatria e Programa de Pós-Graduação em Neuropsiquiatria e Ciências do Comportamento, UFPE.

Recebido em 22/11/2010, aceito em 24/08/2011. Não foram declarados conflitos de interesse associados à publicação deste artigo.

Como citar: Dutra TG, Kurtinaitis LC, Cantilino A, Vasconcelos MC, Hazin I, Sougey EB. A supergeneralização da memória autobiográfica nos transtornos depressivos. Trends Psychiatry Psychother. 2012;34(2):73-9.
} 


\section{Introdução}

A memória autobiográfica relaciona-se à capacidade que as pessoas têm de recordar eventos pessoais e fatos de suas vidas ${ }^{1}$. Pode ser conceituada como um processo reconstrutivo que relaciona a memória episódica e sua característica sensório-perceptual com seu contexto, ou ainda como uma evocação episódica de datas, eventos e acontecimentos pessoais considerando-se a relação temporal e espacial entre eles ${ }^{2}$. A memória autobiográfica também pode ser referida como um processo de memória que combina elementos de memória semântica, pelo fato de que é necessário um conhecimento de si próprio para que os fatos ocorridos sejam significados como pertencentes ao indivíduo, com memória episódica, por serem narrativas pessoais conhecidas pelo sujeito como fazendo parte do seu passado ${ }^{3}$.

Apesar das várias formas de se expressar o conceito de memória autobiográfica, um aspecto peculiar diz respeito ao fato de que a evocação traz consigo a consciência de que um determinado fato ou acontecimento ocorreu anteriormente consigo próprio, fazendo parte de sua história pessoal². Além disso, a evocação apresenta como característica a sensação de reviver o fato, de fazer uma viagem de volta ao passado ${ }^{4}$, chamada por Tulving ${ }^{5}$ de "consciência autonoética". Desta forma, a memória autobiográfica acompanha um senso de re-experiência do evento original e da crença de que ele aconteceu ${ }^{6}$.

Estudos discutem que o desempenho da memória autobiográfica em indivíduos com transtorno depressivo aparece prejudicado em comparação a indivíduos controle sem depressão. Indivíduos com transtornos emocionais - especialmente pacientes com história de depressão - parecem apresentar uma inabilidade de recordar eventos vividos de forma específica, tendendo a uma recuperação supergeneralizada com descrições categóricas?. Quando tentam recuperar eventos específicos, os indivíduos primeiro acessam descrições de eventos gerais, na formação da hierarquia de memórias, utilizando-se de estágios intermediários para seguir sua pesquisa até chegar aos eventos específicos. Administrar essa hierarquia para encontrar o nível de especificidade requerido não representa um problema para a maioria das pessoas. Em alguns casos, todavia, a pesquisa para em estágios intermediários, resultando em uma memória geral em vez de específica, fenômeno que é denominado de memória supergeneralizada.

Conway \& Pleydell-Pearce ${ }^{1}$ propuseram um modelo teórico para a memória autobiográfica, caracterizado por um sistema hierárquico que abrange todos os níveis de conhecimento autobiográfico: períodos de vida, eventos gerais e conhecimentos específicos de eventos. Tais instâncias funcionariam como um banco de dados que estaria modulando a construção das memórias, tanto na codificação quanto na recuperação, e representariam níveis distintos que vão da generalização à especificidade. Num processo de busca, descrições genéricas podem ser inibidas para alcançar um evento específico. Entretanto, se, durante esse processo, não houver recursos executivos suficientes, a recordação específica será prematuramente interrompida, levando à recordação de uma memória geral, com menor grau de especificidade ${ }^{8}$.

A supergeneralização é definida como a propensão a recordar o próprio passado de maneira demasiadamente sintética, genérica e inespecífica ${ }^{9,10}$. Tal fenômeno, no entanto, não está relacionado ao conteúdo da memória, e sim à falta de especificidade espaço-temporal ${ }^{11}$. De forma pioneira, Williams \& Broadbent ${ }^{12}$ investigaram a associação entre recordação congruente e humor em indivíduos que tentaram suicídio. Os autores observaram que, quando comparados com controles, os casos apresentaram uma grande proporção de memórias não específicas e maior latência de resposta em relação a palavras positivas. Uma memória específica foi definida como uma ocasião ou um evento experimentado pessoalmente que aconteceu num espaço de tempo inferior a 1 dia (Williams JM, Autobiographical memory test, manuscrito não publicado) $)^{5}$. Um exemplo de memória específica seria "o dia em que minha mãe morreu". As memórias não específicas ou generalizadas que dizem respeito a eventos repetidos e sem referência a um tempo específico são chamadas de memórias categóricas; já as memórias que remetem a um período de tempo maior, superior a 1 dia, são chamadas de memórias estendidas (Williams JM, Autobiographical memory test, manuscrito não publicado).Um exemplo de memória categórica seria "quando amamentava meu bebê", e um exemplo de memória estendida seria "o tempo que passei em Brasília".

Passados mais de 20 anos, a supergeneralização da memória autobiográfica continua sendo estudada, uma vez que essa tendência de recordação parece estar relacionada a aspectos cognitivos clinicamente importantes, tais como déficits nas habilidades de resolução de problemas ${ }^{13}$, dificuldades para imaginar o futuro ${ }^{14}$ e vulnerabilidade a atos suicidas e parassuicidas ${ }^{15,16}$. O fenômeno da supergeneralização pode ainda estar implicado num pior prognóstico e ser um fator de vulnerabilidade para depressão ${ }^{9,17}$. Estudiosos apontam que o fenômeno pode ser associado a um traço marcador que pode perdurar mesmo após a remissão dos sintomas. Nesse caso, o fenômeno das memórias autobiográficas supergeneralizadas não seria um estado dependente do humor, e sim um traço estável que tornaria indivíduos mais vulneráveis à depressão ou à recaída ${ }^{18}$.

Dessa forma, tal característica é compreendida por alguns pesquisadores como um confiável preditor do 
curso da depressão: pacientes que são mais supergeneralizados em suas evocações parecem continuar preenchendo os critérios para depressão quando testados algumas semanas após o início do tratamento ${ }^{19}$. O primeiro estudo nesse sentido acompanhou pacientes com depressão maior e observou uma relação entre o padrão de evocação supergeneralizado no Teste de Memória Autobiográfica (TMA) e um pior prognóstico, sugerindo que o nível de especificidade permanece estável mesmo quando a gravidade dos sintomas depressivos declina ${ }^{17}$. O TMA ${ }^{12}$ consiste em uma série de palavras-estímulo de diferentes valências emocionais (positiva, neutra e negativa) que são apresentadas a um determinado indivíduo. Para cada palavra apresentada, o entrevistado deve procurar recordar eventos específicos de sua história de vida. No Brasil, o teste foi adaptado por Pergher ${ }^{20}$ e subsequentemente avaliado por Pergher \& Stein ${ }^{21}$.

Em pouco mais de 20 anos, desde o estudo inicial de Williams \& Broadbent ${ }^{12}$, o interesse pela relação entre a especificidade da memória autobiográfica e a psiquiatria tem aumentado, uma vez que a supergeneralização parece apresentar-se como uma característica de alta relevância clínica e teórica no que concerne à vulnerabilidade para a depressão e para outros transtornos emocionais. Desta forma, a investigação de tal fenômeno contribui para a elucidação de mecanismos neurocognitivos subjacentes que podem potencializar o caráter crônico de certas psicopatologias ${ }^{22}$.

Diante do exposto, o objetivo deste trabalho foi revisar estudos que focaram a temática da supergeneralização da memória autobiográfica e discutir seus principais achados relacionados à supergeneralização e aos transtornos depressivos.

\section{Método}

Foi realizada um revisão sistematizada em busca de artigos nas bases de dados LILACS, SciELO, MEDLINE, IBECS e nas bases de ciências da saúde do Portal de Periódicos da Coordenação de Aperfeiçoamento de Pessoal de Nível Superior (CAPES). A pesquisa focou artigos publicados entre os anos de 2000 e 2010. Os seguintes descritores foram utilizados: memória autobiográfica, supergeneralização da memória autobiográfica e memória autobiográfica e depressão, em português; e autobiographical memory, overgeneral autobiographical memory e autobiographical memory and depression em inglês. Foram considerados também os seguintes critérios de inclusão: artigos originais, que usassem o TMA como instrumento de investigação, e que tivessem como interesse o estudo da relação entre a supergeneralização da memória autobiográfica e os transtornos depressivos.
Foram captados 86 artigos, dos quais 59 foram excluídos por não cumprirem os critérios de inclusão propostos. Assim, no total, 27 estudos foram analisados.

\section{Resultados e discussão}

Os dados principais dos estudos revisados são apresentados na Tabela 1.

Uma discussão relevante em relação à supergeneralização da memória autobiográfica diz respeito à questão de que o fenômeno parece estar relacionado à depressão, sua manutenção e prognóstico. Verificou-se, por exemplo, que estudantes que se apresentaram supergeneralizados em suas respostas e estavam submetidos a eventos estressantes de vida relataram mais sintomas depressivos num período de 4 a 6 semanas. Isso sugere que a supergeneralização da memória constitui um fator de vulnerabilidade cognitiva que predispõe indivíduos ao desenvolvimento de transtorno emocional quando expostos a eventos de vida difíceis ${ }^{9}$. Em outro estudo, indivíduos que foram testados ao iniciar o tratamento da depressão e mostraram mais supergeneralização tiveram uma significativa chance de preencher os critérios diagnósticos quando retestados após algumas semanas de tratamento, mostrando que a investigação da memória autobiográfica pode ser um preditor de resposta ao tratamento ${ }^{19}$. Na mesma linha, quando a depressão foi controlada em indivíduos diagnosticados, a redução da especificidade para estímulos negativos predisse um prognóstico pior, levando-se em consideração também que a relação entre um estilo de pensamento ruminativo e a supergeneralização pode representar aspectos diferentes de um processo cognitivo comum subjacente, e que tais características exerceriam efeito negativo sobre o curso do transtorno ${ }^{34}$.

Em outro estudo, pacientes em estado eutímico também apresentaram prejuízo na especificidade, sugerindo que tal característica não seria apenas um epifenômeno cognitivo dependente do humor, e sim um déficit neurocognitivo geral de indivíduos que já passaram por episódio de depressão maior, mesmo que não haja uma associação entre tal característica e o número de recaídas, de episódios prévios ou sintomas residuais ${ }^{37}$. Em se tratando de indivíduos portadores de depressão sazonal e deprimidos durante o inverno, observou-se que o aumento da supergeneralização para palavras-estímulo positivas relacionou-se com a intensidade de sintomas no verão, em estado de remissão. Os resultados sugeriram também que a geração das memórias generalizadas parece apresentar-se como um processo psicológico importante no que diz respeito ao curso da depressão ${ }^{47}$. A supergeneralização também foi verificada na depressão após o 
Tabela 1 - Características dos artigos incluídos na revisão

\begin{tabular}{|c|c|c|c|c|}
\hline Estudo & Ano & Tema & $\begin{array}{c}\text { Média } \\
\text { de idade }\end{array}$ & $\begin{array}{c}\text { Total de participantes } \\
\text { (homem/mulher) }\end{array}$ \\
\hline Croll \& Bryant ${ }^{23}$ & 2000 & Depressão pós-parto & 31,85 & $0 / 26$ \\
\hline Mackinger et al. ${ }^{24}$ & 2000 & Mudanças afetivas no pós-parto & 29,3 & $0 / 50$ \\
\hline Watkins et al. ${ }^{25}$ & 2000 & Depressão maior & 39,5 & $16 / 32$ \\
\hline Williams et al. ${ }^{26}$ & 2000 & Depressão maior & 44,0 & $22 / 33$ \\
\hline Barnhofer et al. ${ }^{27}$ & 2002 & Depressão maior & 29,4 & $3 / 12$ \\
\hline Burnside et al. ${ }^{28}$ & 2004 & Depressão maior/trauma & 36,74 & $0 / 50$ \\
\hline Gibbs \& Rude ${ }^{9}$ & 2004 & Depressão maior & 21,23 & $32 / 49$ \\
\hline Iqbal et al. ${ }^{29}$ & 2004 & Depressão pós-psicótica & 25,0 & $29 *$ \\
\hline Kremers et al. ${ }^{30}$ & 2004 & Depressão maior/transtorno de personalidade borderline & 37,1 & $17 / 122$ \\
\hline Kaviani et al. ${ }^{15}$ & 2005 & Depressão maior/comportamento suicida & 27,85 & $40 *$ \\
\hline Renneberg et al. ${ }^{31}$ & 2005 & Depressão maior/transtorno de personalidade borderline & 32,0 & $0 / 87$ \\
\hline Drummond et al. ${ }^{32}$ & 2006 & Disforia infantil & 9,23 & $35 / 35$ \\
\hline Kuyken et al. ${ }^{33}$ & 2006 & Depressão maior/trauma & - & $62 *$ \\
\hline Raes et al. ${ }^{34}$ & 2006 & Depressão maior & 40,2 & $9 / 19$ \\
\hline Rekart et al. ${ }^{35}$ & 2006 & Disforia & - & $17 / 39$ \\
\hline Roberts et al. ${ }^{36}$ & 2006 & Sintomas depressivos/autoestima & 19,8 & $92 / 112$ \\
\hline Spinhoven et al. ${ }^{37}$ & 2006 & Depressão maior & 44,7 & $122 *$ \\
\hline Crane et al. ${ }^{38}$ & 2007 & Ruminação depressiva/depressão maior & 31,0 & $11 / 23$ \\
\hline Heidenreich et al. ${ }^{39}$ & 2007 & Depressão maior/fobia social & 34,8 & $24 / 30$ \\
\hline Serrano et al. ${ }^{40}$ & 2007 & Sintomas depressivos & 72,32 & 77.85 \\
\hline \multirow[t]{2}{*}{ Spinhoven et al. ${ }^{41}$} & 2007 & Atitudes disfuncionais/depressão & $44,4^{+}$ & $111^{*}$ \\
\hline & & Transtorno de personalidade borderline & 30,8 & $82 *$ \\
\hline Sutherland \& Bryant ${ }^{42}$ & 2007 & Ruminação depressiva/depressão maior & 20,82 & $19 / 35$ \\
\hline Vrielynck et al. ${ }^{43}$ & 2007 & Depressão infantil & 11,5 & $27 / 13$ \\
\hline Bessell et al. ${ }^{44}$ & 2008 & Ruminação de depressiva/dano cerebral adquirido & - & $42 / 16$ \\
\hline Hermans et al. ${ }^{19}$ & 2008 & Depressão maior & 39,9 & $6 / 14$ \\
\hline Rasmussen et al. ${ }^{45}$ & 2008 & Comportamento suicida & 38,0 & $17 / 23$ \\
\hline Schlosser et al. ${ }^{46}$ & 2010 & Depressão maior & 34,1 & $16 / 16$ \\
\hline
\end{tabular}

* Artigos que não explicitam o número de participantes separados por gênero.

+ Artigos que incluem dois estudos no seu texto.

primeiro episódio psicótico, verificando-se que pacientes deprimidos recordaram mais memórias generalizadas do que pacientes não deprimidos, particularmente em relação às evocações com palavras-estímulo positivas; no entanto, não houve diferenças entre os grupos quanto à latência de recordação' ${ }^{29}$.

Deprimidos também foram comparados com indivíduos que possuíam transtorno de personalidade borderline e controles. Os resultados corroboraram a literatura no tocante à relação entre depressão e supergeneralização ${ }^{31}$. Os deprimidos apresentaram dificuldade em recordar memórias específicas, maior latência para a recuperação e alta proporção de recordações categóricas. Os indivíduos borderline não diferiram dos controles na especificidade, e observou-se que, em relação aos deprimidos, aqueles apresentaram um acesso relativamente fácil e rápido a memórias com valência negativa. Tal característica pode ser específica para esse grupo e oposta aos achados na depressão ${ }^{36}$, que parece instalar nos processos cognitivos dos indivíduos dificuldades para atingir a especificidade, recuperando consecutivamente memórias categóricas ${ }^{27}$. Ao se compararem pacientes borderline depressivos, pacientes borderline não depressivos e controles, também se verificou que os borderline não depressivos não diferiram dos controles, enquanto que os borderline com depressão recordaram menos memórias específicas ${ }^{30}$. Contrariamente ao estudo de Renneberg et al. ${ }^{31}$, tanto indivíduos depressivos quanto borderline recuperaram menos memórias específicas, encontrando-se uma relação entre palavras-estímulo e esquemas cognitivos/atitudes disfuncionais ${ }^{41}$. Em divergência com o corpo de estudos que enfocam a depressão, uma comparação entre pacientes deprimidos, pacientes com fobia social e controles não demonstrou diferenças significativas em relação à habilidade de recuperar memórias específicas entre os três grupos distintos, destacando-se apenas que, quanto maior o nível de educação dos participantes, mais memórias específicas foram recuperadas ${ }^{39}$.

Diante da hipótese de regulação do afeto de Willia$\mathrm{ms}^{7}$, na qual a supergeneralização se desenvolveria como um mecanismo de evitação de memórias dolorosas, a relação entre abuso sexual, desenvolvimento da depressão e supergeneralização já foi estudada28,33. Observou-se um maior número de respostas categóricas dadas por mulheres que tinham experimentado abuso em uma idade menor ou por maior duração de tempo. No entan- 
to, mulheres que não relataram depressão foram mais categóricas em relação àquelas que reportaram episódio depressivo na idade adulta, levantando a questão de que a supergeneralização pode estar mais associada a um fator de proteção do que de vulnerabilidade, sendo, mesmo assim, um mecanismo de regulação afetiva ${ }^{28}$. Entre adolescentes que foram expostos a diferentes formas de abuso, observou-se, entre aqueles que atingiram o ponto de corte para depressão, maior produção de memórias genéricas ou supergeneralizadas quando comparados aos adolescentes não deprimidos. Os mais velhos foram menos específicos do que os mais novos, particularmente em resposta a estímulos neutros e negativos ${ }^{33}$.

Os estudos de supergeneralização também alcançaram as crianças. Vrielynch et al. ${ }^{43}$ observaram que, ao se comparar crianças deprimidas com não deprimidas, a recordação das primeiras foi menos específica. As memórias apresentaram-se estendidas, e as omissões foram mais frequentes, independentemente da valência do estímulo proposto. Além disso, quando crianças deprimidas foram comparadas com as que possuíam outros transtornos psiquiátricos e sem histórico de qualquer dificuldade no desenvolvimento, as deprimidas também recuperaram significativamente menos memórias específicas. Isso levantou a discussão de que o fenômeno da supergeneralização não deve ser explicado por um status psiquiátrico geral ou histórico de dificuldades no desenvolvimento.

Os resultados também indicaram que o humor deprimido não media o efeito da supergeneralização, coadunando-se com a visão de que a recuperação supergeneralizada pode representar um traço estável de vulnerabilidade para a depressão, independentemente do curso do transtorno. Crianças mais velhas também pareceram ser mais específicas que as mais novas, independentemente da valência dos estímulos. A disforia na infância também se relacionou com a diminuição da especificidade de memórias a estímulos positivos ${ }^{32}$. Da mesma forma, a disforia foi relacionada à supergeneralização em mulheres jovens submetidas a repetidas tentativas de recuperação mnemônica, destacando-se como fatores relacionados os sintomas somáticos e a baixa autoestima ${ }^{36}$. Outra investigação, comparando estudantes disfóricos com controles, indicou que a ocorrência de um padrão de respostas categóricas também se encontra presente em níveis subclínicos de depressão ${ }^{35}$. Por outro lado, avaliando-se adultos idosos, verificou-se que tanto os deprimidos quanto os não deprimidos tenderam a responder com memórias não específicas; entretanto, observou-se que os deprimidos apresentaram mais supergeneralização para palavras negativas do que para palavras positivas, além de maior latência de respostas. $O$ aumento na latência ocorreu em relação a todos os tipos de evocação, e não apenas às evocações específicas ${ }^{40}$.
Mulheres foram investigadas também quando portadoras de depressão ou alterações afetivas no período pós-parto 23,24 . Os achados apoiam a hipótese de que a supergeneralização pode ser um preditor de alterações afetivas em mulheres grávidas avaliadas durante a $25^{\mathrm{a}}$ e a $40^{\mathrm{a}}$ semana de gestação e reavaliadas após o nascimento dos seus filhos ${ }^{24}$. Além disso, investigando-se mulheres com depressão pós-parto e comparando-as com controles, encontrou-se que as deprimidas recordaram menos memórias específicas, tiveram latência maior de respostas e uma associação entre a gravidade do transtorno e o déficit no acesso à especificidade ${ }^{23}$.

Estudos também focaram a contribuição do pensamento ruminativo na depressão e sua relação com a perda de especificidade ${ }^{19,25,38,42,44}$, embora nem todos os resultados apontem uma relação significativa ${ }^{19}$. Indivíduos com histórico depressivo, quando induzidos experimentalmente a um modo analítico de processamento e testados antes e 8 minutos após a manipulação experimental, apresentaram um declínio na especificidade da memória e níveis mais altos de ruminação. Isso levantou a questão de que indivíduos assintomáticos podem reinstalar um estilo analítico e ruminativo focado em si mesmos, e a baixa especificidade pode ser uma função desse tipo de processamento cognitivo, aumentando a vulnerabilidade a uma subsequente recaída ${ }^{38}$. Outro estudo que comparou pacientes com altos e baixos níveis de depressão observou que, diante da indução a um estilo ruminativo, houve um aumento nas memórias supergeneralizadas em relação aos participantes com alto nível de depressão, não se verificando o mesmo naqueles com baixos níveis depressivos. Em outra etapa do mesmo estudo, os participantes foram induzidos a uma tarefa de ruminação que possuiu diferentes valências - positiva, neutra e negativa -, e observou-se que a indução à ruminação com valência negativa levou a mais memórias supergeneralizadas nos indivíduos com altos níveis de depressão. 0 efeito não foi observado nos indivíduos com baixos níveis de sintomatologia, sugerindo-se que estes podem ter respondido à ruminação negativa com mais estratégias adaptativas e, consequentemente, menor supergeneralização. De forma geral, a ruminação negativa foi entendida como um mecanismo mediador entre a recordação de memórias supergeneralizadas e a depressão ${ }^{42}$.

Resultados nessa mesma linha foram encontrados também ao se investigar indivíduos que possuíam dano cerebral adquirido e eram vulneráveis à depressão. Induzidos a condições de ruminação e distração, e reavaliados em relação à evocação de memórias específicas, a ruminação significativamente aumentou a proporção de memórias supergeneralizadas, comparando-se os estados pré e pós-intervenção ${ }^{44}$. Padrão semelhante foi encontrado em indivíduos depressivos ou disfóricos submetidos a manipu 
lação com o mesmo fim ${ }^{25}$. Quando pacientes depressivos foram testados em relação a várias tarefas de memória e ruminação, encontrou-se também que a supergeneralização esteve relacionada à diminuição da capacidade de memória de trabalho e à ruminação, mas não ao desempenho de memória semântica e episódica. Tais dados sugerem que a supergeneralização não é meramente um déficit de memória geral vinculado à depressão ${ }^{19}$.

Em consonância com os estudos que possuem como foco os aspectos cognitivos, foram investigados indivíduos que apresentaram comportamento suicida e a relação dessa característica com traços perfeccionistas. Observou-se que indivíduos que tinham investido contra si mesmos repetidas vezes foram mais supergeneralizados em suas recordações para os estímulos positivos, e um padrão de perfeccionismo social esteve relacionado com a supergeneralização e altos níveis de ideação suicida. Nesse sentido, a integração de traços de personalidade e características cognitivas parece proporcionar um melhor conhecimento sobre o suicídio e comportamentos autodestrutivos ${ }^{45}$. Em outro estudo, pacientes que tentaram suicídio e preencheram os critérios para depressão maior também produziram mais memórias supergeneralizadas, apresentando maior latência para palavras-estímulo positivas quando comparados com controles e mais estratégias de resolução de problemas escassas e menos efetivas ${ }^{15}$.

Dentro de uma perspectiva neuroendócrina, a especificidade da memória autobiográfica foi observada em relação ao nível de cortisol em pacientes com depressão maior ${ }^{46,48}$. Contrariamente à expectativa do estudo, encontrou-se que o aumento do nível de cortisol esteve relacionado a um melhor desempenho de evocação em relação à especificidade, enquanto que a redução foi associada com respostas mais categóricas ${ }^{48}$. De outra forma, examinando-se o impacto da administração aguda de cortisol sobre o desempenho da memória autobiográfica em pacientes com depressão maior comparados com controles, observou-se que o cortisol não reduziu o desempenho da evocação nos deprimidos, como verificado nos controles. É possível que uma redução da sensibilidade do receptor do glicocorticoide nos deprimidos esteja associada a tal resultado ${ }^{46}$.

Diante da relação estudada entre a supergeneralização e a depressão, outra vertente procurou investigar se a memória autobiográfica poderia ser sensível a tratamento psicológico. Indivíduos que já tinham passado por episódio depressivo foram avaliados antes e depois de passar por um protocolo de terapia cognitiva com o objetivo de intervir sobre as memórias autobiográficas. Os resultados destacaram uma diminuição da generalidade em suas respostas em relação a um grupo com igual diagnóstico que não foram submetidos ao protocolo. Nesse sentido, embora tal déficit de memória possa ser um estilo cognitivo construído, ele se apresentou como passível de modificação ${ }^{26}$.

\section{Conclusão}

A revisão realizada apresenta um conjunto de pesquisas que têm estudado o fenômeno da supergeneralização da memória autobiográfica e sua relação com transtornos depressivos. O interesse pela supergeneralização da memória autobiográfica vem adquirindo maior interesse da comunidade científica, principalmente no que diz respeito à investigação de sua relação com transtornos do humor. No que concerne à investigação de indivíduos depressivos, a maior parte dos estudos enfoca o déficit de especificidade em comparação a controles, mesmo quando a sintomatologia depressiva apresenta remissão, sugerindo que tal estilo cognitivo parece se tornar uma característica estável, o que pode ter implicações no prognóstico dos transtornos afetivos e estar relacionado a características psicopatológicas como o fluxo ruminativo de pensamentos na depressão.

Destaca-se, entretanto, que, apesar de algumas pesquisas ressaltarem tal fenômeno como um traço cognitivo estável, a utilização de métodos psicoterápicos focados na supergeneralização pode proporcionar mudanças relacionadas ao aumento da especificidade. Uma vez que a supergeneralização tem sido associada a prejuízo na resolução de problemas, na imaginação do futuro e na regulação afetiva, considerar tal característica como implicada na esfera cognitiva dos transtornos afetivos pode gerar uma melhor compreensão de padrões cognitivos que podem reforçar um processamento disfuncional relacionado à manutenção de tais transtornos. No entanto, como alguns estudos apresentam resultados divergentes, faz-se necessário o desenvolvimento de mais estudos, principalmente de caráter longitudinal, que possibilitem dirimir ou tentar explicar mais claramente as inconsistências ainda existentes.

\section{Referências}

1. Conway MA, Pleydell-Pearce CW. The construction of autobiographical memories in the self-memory system. Psychol Rev. 2000;107:261-88.

2. Greenberg DL, Rubin DC. The neuropsychology of autobiographical memory. Cortex. 2003;39:687-728.

3. Frank J, Landeira-Fernandez J. Rememoração, subjetividade e as bases neurais da memória autobiográfica. Psic Clin. 2006; $18: 35-47$.

4. Wheeler MA, Stuss DT, Turving E. Toward a theory of episodic memory: the frontal lobes and autonoetic consciousness. Psychol Bull. 1997;121:331-54.

5. Tulving E. Memory and consciousness. Can Psychol. 1985;26:1-10.

6. Gauer G, Gomes WG. A experiência de recordar em estudos da memória autobiográfica: aspectos fenomenais e cognitivos. Memorandum. 2006;11:102-12.

7. Williams JM. Depression and the specificity of autobiographical memory. In: Rubin DC, editor. Remembering our past: studies in autobiographical memory. Cambridge: Cambridge University Press; 1996. p. 244-67. 
8. Haque S, Conway MA. Sampling the processes of autobiographical memory construction. Eur J Cognit Psychol. 2001;13:529-47.

9. Gibbs BR, Rude SS. Overgeneral autobiographical memory as depression vulnerability. Cognit Ther Res. 2004;28:511-26.

10. Pergher, GK, Oliveira RG, De Ávila LM, Stein L M. Memória, humor e emoção. Rev Psiquiatr Rio Gd Sul. 2006;28:61-8.

11. Peeters F, Merckelbach H, Boon-Vermeeren M. Autobiographical memory specificity and the course of major depressive disorder. Compr Psychiatry. 2002;43:344-50.

12. Williams JM, Broadbent K. Autobiographical memory in suicide attempters. J Abnorm Psychol. 1986;95:144-9.

13. Goddard L, Drithchel B, Burton A. Role of autobiographical memory in social problem solving and depression. J Abnorm Psychol. 1996;105:609-16.

14. Williams JM, Ellis NC, Tyers C, Healy H, Rose G, Macleod AK. The specificity of autobiographical memory and imageability of the future. Mem Cognit. 1996;24:116-25.

15. Kaviani H, Rahimi-Darabad P, Naghavi HR. Autobiographical memory retrieval and problem-solving deficits of Iranian depressed patients attempting suicide. J Psychopathol Behav Assess. 2005;27:39-44.

16. Pollock LR, Williams JM. Effective problem solving in suicide attempters depends on specificity autobiographical recall. Suicide Life Threat Behav. 2001;31:386-96

17. Brittkebank JS, Williams JM, Ferrier IN. Autobiographical memory in depression: state or trait marker? Br J Clin Psychol. $1993 ; 162: 118-21$.

18. Raes F, Watkins E, Williams JM, Hermans D. Non-ruminative processing reduces autobiographical memory retrieval in students. Behav Res Ther. 2008;46:748-56.

19. Hermans D, Vandromme H, Debeer $E$, Raes F, Demyttenaere K, Brunfaut $E$, et al. Overgeneral autobiographical memory predicts diagnostic status in depression. Behav Res Ther. 2008;46:668-77.

20. Pergher GK. Avaliando a especificidade da memória autobiográfica: o teste de memória autobiográfica [dissertação]. Porto Alegre: Pontifícia Universidade Católica do Rio Grande do Sul; 2005.

21. Pergher GK, Stein LM. Recuperando memórias autobiográficas: avaliação da versão brasileira do Teste de Memória Autobiográfica. Psico. 2008;39:299-307.

22. Raes F, Hermans D, Philippot, P, Kremers I. Autobiographical memory specificity and psychopathology: the broader context of cognition and emotion research. Cogn Emot. 2006;20:324-27.

23. Croll S, Bryant R. Autobiographical memory in postnatal depression. Cognit Ther Res. 2000;24:419-26.

24. Mackinger HF, Loschin GG, Leibetseder MM. Prediction of postnatal affective changes by autobiographical memories. Eur Psychol. 2000;5:52-61.

25. Watkins E, Teasdale JD, Williams RM. Decentring and distraction reduce overgeneral autobiographical memory in depression. Psychol Med. 2000;30:911-20.

26. Williams JM, Teasdale JD, Segal ZV, Soulsby J. Mindfulnessbased cognitive therapy reduces overgeneral autobiographical memory in formerly depressed patients. J Abnorm Psychol. 2000;109:150-5.

27. Barnhofer T, Jong-Meyer R, Kleipab A, Nikesch S. Specificity of autobiographical memories in depression: an analysis of retrieval processes in a think-aloud task. Br J Clin Psychol. 2002;41:411-6.

28. Burnside E, Startup M, Byatt M, Rollinson L, Hill J. The role of overgeneral memory in the development of adult depression following childhood trauma. Br J Clin Psychol. 2004;43:365-73.

29. Iqbal Z, Birchwood M, Hemsley D, Jackson C, Morris E. Autobiographical memory and post-psychotic depression in first episode psychosis. Br J Clin Psychol. 2004;43:97-104.

30. Kremers IP, Spinhoven PH, Van der Does AJ. Autobiographical memory in depressed and non-depressed patients with bor- derline personality disorder. Br J Clin Psychol. 2004;43:17-29.

31. Renneberg B, Theobald E, Nobs M, Weisbrod M. Autobiographical memory in borderline personality disorder and depression. Cognit Ther Res. 2005;29:343-58.

32. Drummond LE, Dritschel B, Astell A, O' Carroll RE, Dalgleish T. Effects of age, dysphoria, and emotion-focusing on autobiographical memory specificity in children. Cogn Emot. 2006;20:488-505

33. Kuyken W, Howell R, Dagleish T. Overgeneral autobiographical memory in depressed adolescents with versus without a reported history of trauma. J Abnorm Psychol. 2006; 115:387-96.

34. Raes F, Hermans D, Williams JM, Beyers W, Brunfaunt E. Reduced autobiographical memory specificity and rumination in predicting the course of depression. J Abnorm Psychol. 2006; 115:699-704.

35. Rekart KN, Mineka S, Zinbarg RE. Autobiographical memory in dysphoric and non-dysphoric college students using a computerized version of the AMT. Cogn Emot. 2006;20:506-15.

36. Roberts JE, Carlos EL, Kashdan TB. Impact of depressive symptoms, self-esteem and neuroticism on trajectories of overgeneral autobiographical memory over repeated trials. Cogn Emot. 2006;20:383-401.

37. Spinhoven $P$, Bockting CL, Koeter MW, Wekking EM, Williams JM. Autobiographical memory in the euthymic phase of recurrent depression. J Abnorm Psychol. 2006;115:590-600.

38. Crane C, Barnhofer T, Visser C, Nightingale H, Williams JM. The effects of analytical and experimental rumination on autobiographical memory specificity in individuals with a history of major depression. Behav Res Ther. 2007;45:3077-87.

39. Heidenreich T, Jughanns-Royack K, Stangier U. Specificity of autobiographical memory in social phobia and major depression. Br J Clin Psychol. 2007;46:19-33.

40. Serrano JP, Latorre JM, Gatz M. Autobiographical memory in older adults with and without depressive symptoms. Int J Clin Health Psychol. 2007;7:41-57.

41. Spinhoven $P$, Bockting $C L$, Kremers IP, Schene AH, Williams JM. The endorsement of dysfunctional attitudes is associated with an impaired retrieval of specific autobiographical memories in response to matching cues. Memory. 2007;15:324-38.

42. Sutherland K, Bryant RA. Rumination and overgeneral autobiographical memory. Behav Res Ther. 2007;45:2407-16.

43. Vrielynck N, Deplus S, Philippot P. Overgeneral autobiographical memory in depressive disorder in children. J Clin Adolesc Psychol. 2007;36:95-105.

44. Bessel AL, Watkins ER, Williams WH. Depressive rumination reduces specificity of autobiographical memory recall in acquired brain injury. J Int Neuropsychol Soc. 2008;14:63-70.

45. Rasmussen SA, O'Connor RC, Brodie D. The role of perfectionism and autobiographical memory in a sample of parasuicide patients: an exploratory study. Crisis. 2008;29:64-7.

46. Schlosser N, Wolf OT, Fernando SC, Riedsel K, Otte C, Muhtz C, et al. Effects of acute cortisol administration on autobiographical memory in patients with major depression and healthy controls. Psychoneuroendocrinology. 2010;35:316-20.

47. Dagleish T, Spinks H, Yiend J, Kuyken W. Autobiographical memory style in seasonal affective disorder and its relationship to future symptom remission. J Abnorm Psychol. 2001; 110:335-40.

48. Banhofer T, Kuehn EM, Jong-Meyer R. Specificity of autobiographical memories and basal cortisol levels in patients with major depression. Psychoneuroendocrinology. 2005;30:403-11.

\section{Correspondência:}

Tarcísio Gomes Dutra

Rua Sete de Setembro, 315, Boa Vista

55644-150 - Gravatá, PE

E-mail: tgdutra@yahoo.com.br 